\title{
Apex of the Prostate
}

National Cancer Institute

\section{Source}

National Cancer Institute. Apex of the Prostate. NCI Thesaurus. Code C13087.

The lowest part of the prostate gland adjacent to the superior fascia of the urogenital diaphragm. 